\title{
Radiation Exposure to the Hand of a Spinal Interventionalist during Fluoroscopically Guided Procedures
}

\author{
Kazuta Yamashita ${ }^{1}$, Hisanori Ikuma ${ }^{2}$, Takuya Tokashiki ${ }^{2}$, Takashi Maehara ${ }^{2}$, \\ Akihiro Nagamachi ${ }^{1}$, Yoichiro Takata ${ }^{1}$, Toshinori Sakai ${ }^{1}$, Kosaku Higashino ${ }^{1}$, Koichi Sairyo ${ }^{1}$ \\ ${ }^{I}$ Department of Orthopedics, Institute of Biomedical Sciences, Tokushima University Graduate School, Tokushima, Japan \\ ${ }^{2}$ Department of Orthopedics, Kagawa Rosai Hospital, Marugame, Japan
}

\section{Study Design: Prospective study.}

Purpose: During fluoroscopically guided spinal procedure, the hands of spinal surgeons are placed close to the field of radiation and may be exposed to ionizing radiation. This study directly measured the radiation exposure to the hand of a spinal interventionalist during fluoroscopically guided procedures.

Overview of Literature: Fluoroscopically guided spinal procedures have been reported to be a cause for concern due to the radiation exposure to which their operators are exposed.

Methods: This prospective study evaluated the radiation exposure of the hand of one spinal interventionalist during 52 consecutive fluoroscopic spinal procedures over a 3-month period. The interventionalist wore three real-time dosimeters secured to the right forearm, under the lead apron over the chest, and outside the lead apron over the chest. Additionally, one radiophotoluminescence glass dosimeter was placed under the lead apron over the left chest and one ring radiophotoluminescence glass dosimeter was worn on the right thumb. The duration of exposure and radiation dose were measured for each procedure.

Results: The average radiation exposure dose per procedure was $14.9 \mu \mathrm{Sv}, 125.6 \mu \mathrm{Sv}$, and $200.1 \mu \mathrm{Sv}$, inside the lead apron over the chest, outside the lead apron over the chest, and on the right forearm, respectively. Over the 3-month period, the protected radiophotoluminescence glass dosimeter over the left chest recorded less than the minimum reportable dose, whereas the radiophotoluminescence glass ring dosimeter recorded $368 \mathrm{mSv}$ for the thumb.

Conclusions: Our findings indicated that the cumulative radiation dose measured at the dominant hand may exceed the annual dose limit specified by the International Commission on Radiological Protection. Spinal interventionalists should take special care to limit the duration of fluoroscopy and radiation exposure.

Keywords: Radiation exposure; Spinal procedure; Fluoroscopy; Hand; Fingers

\section{Introduction}

Over time, fluoroscopy has been overused and abused. It is well known that radiation carries certain risks to those who are exposed. Long-term exposure to ionizing radiation has been linked to numerous malignancies, genetic

\footnotetext{
Received May 31, 2016; Revised Jun 8, 2016; Accepted Jun 26, 2016

Corresponding author: Kazuta Yamashita

Department of Orthopedics, Institute of Biomedical Sciences, Tokushima University Graduate School,

3-18-15 Kuramoto-cho, Tokushima 770-8503, Japan

Tel: +81-88-633-7240, Fax: +81-88-633-0178, E-mail: kazutayamasita0311@hotmail.com
} 
and hematological aberrations, dermatological lesions, and cataracts [1-6]. Recent years have witnessed a growing awareness of the harmful effects of long-term exposure to low-dose irradiation. Mastrangelo et al. [7] reported a significant increase in the incidence of tumors among orthopedic surgeons over a 14-year period. Further, Chou et al. $[8,9]$ confirmed that the prevalence of cancer and breast cancer among female orthopedic surgeons is 1.9 -fold and 2.9-fold higher than that in the general U.S. female population, respectively. This elevated risk of cancer has been partly attributed to continuous exposure to ionizing radiation [10].

In general, orthopedic surgeons lack awareness regarding radiation exposure and its health effects and are careless about taking the required protective measures. While performing fluoroscopic procedures, most orthopedic surgeons use a lead apron-which covers only the abdomen and genitals-for protection. However, the hands and fingers of orthopedic surgeons, which tend to be placed close to the procedure field, remain susceptible to direct exposure to ionizing radiation (Fig. 1). The surgeon's hands may be at greater risk for radiation exposure than previously recognized [11-13]. Few studies have assessed the radiation exposure of spinal interventionalists performing fluoroscopically guided procedures, such as

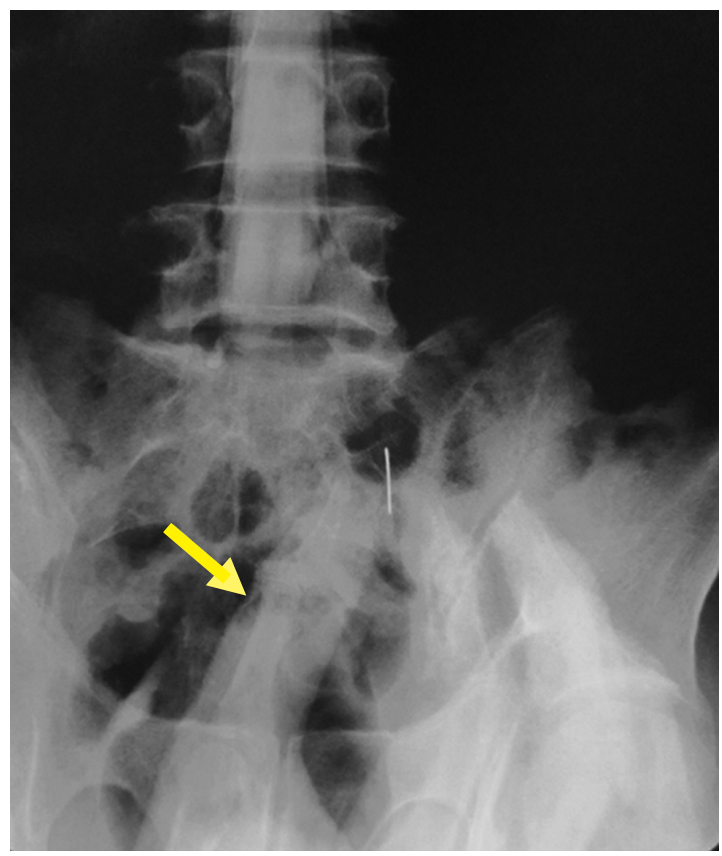

Fig. 1. The hand of the spinal interventionalist is directly exposed to ionizing radiation during right S1 nerve root block. Arrow shows the right thumb of the spinal interventionalist. myelography, selective nerve root block, and facet joint block.

In this study, we sought to directly measure the radiation exposure of the hand and fingers of one spinal interventionalist during fluoroscopically guided procedures in a series of consecutive cases to determine whether the exposure levels were within the dose limits set by the International Commission on Radiological Protection (ICRP) [14]. In addition, we measured the effective whole-body dose of radiation that the spinal interventionalist is exposed to during the abovementioned procedures and the effectiveness of the lead apron in preventing this exposure.

\section{Materials and Methods}

This study was a prospective investigation aimed at measuring the amount of radiation a spinal interventionalist is exposed to during routine spinal procedures. We conducted a 3-month prospective study between August 1, 2012 and October 31, 2012. One surgeon (K.Y., with 9 years of experience as a spine surgeon) was monitored during 52 consecutive fluoroscopic spine procedures, which included myelography, selective nerve root block, and facet joint block.

During each procedure, the spinal interventionalist wore the same thyroid lead collar and lead apron, which had a lead equivalence of $0.35 \mathrm{~mm}$. During each procedure, five radiation dosimeters were used. One real-time dosimeter (ALOKA, Tokyo, Japan) (Fig. 2A) was secured to each of the dominant right forearm (unprotected) (Fig. $2 \mathrm{~B})$, inside the lead apron over the left chest (protected) (Fig. 2C), and outside the lead apron over the left chest (unprotected) (Fig. 2D). In addition, one radiophotoluminescence glass dosimeter (ALOKA) (Fig. 3A) was placed inside the lead apron over the left chest (protected) to measure the effective whole-body radiation dose (Fig. 3B) and one ring radiophotoluminescence glass dosimeter was worn on the right thumb under the sterile surgical glove (unprotected) (Fig. 3C, D). At monthly intervals, the radiophotoluminescence glass dosimeters were forwarded to the supplier for quantitative analysis.

All procedures were performed using the same fluoroscopic device (FLUOREX Winscope6000, TOSHIBA, Tokyo, Japan) in the automatic mode. The machine was calibrated every 3 months. During all procedures, the spinal interventionalist stood on the left side of the X-ray table, and the X-ray beam source was placed over the X- 

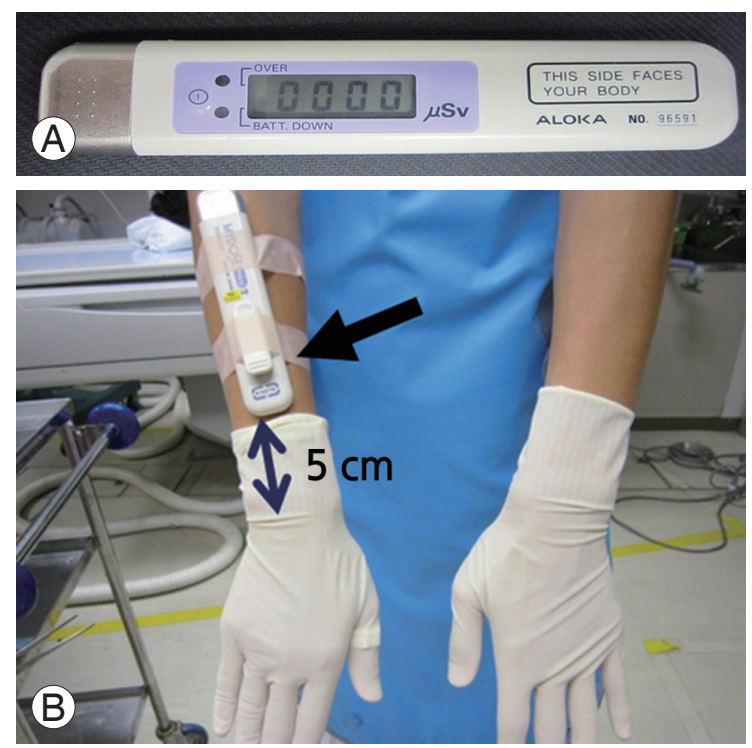

Fig. 2. Positions of the real-time dosimeter (A) exposed to radiation during fluoroscopy. The real-time dosimeter was secured to the spinal interventionalist's right forearm (at a point 5 $\mathrm{cm}$ proximal to the ulnar styloid process) (B), on the left chest under the lead apron (C), and on the left chest over the lead apron (D).
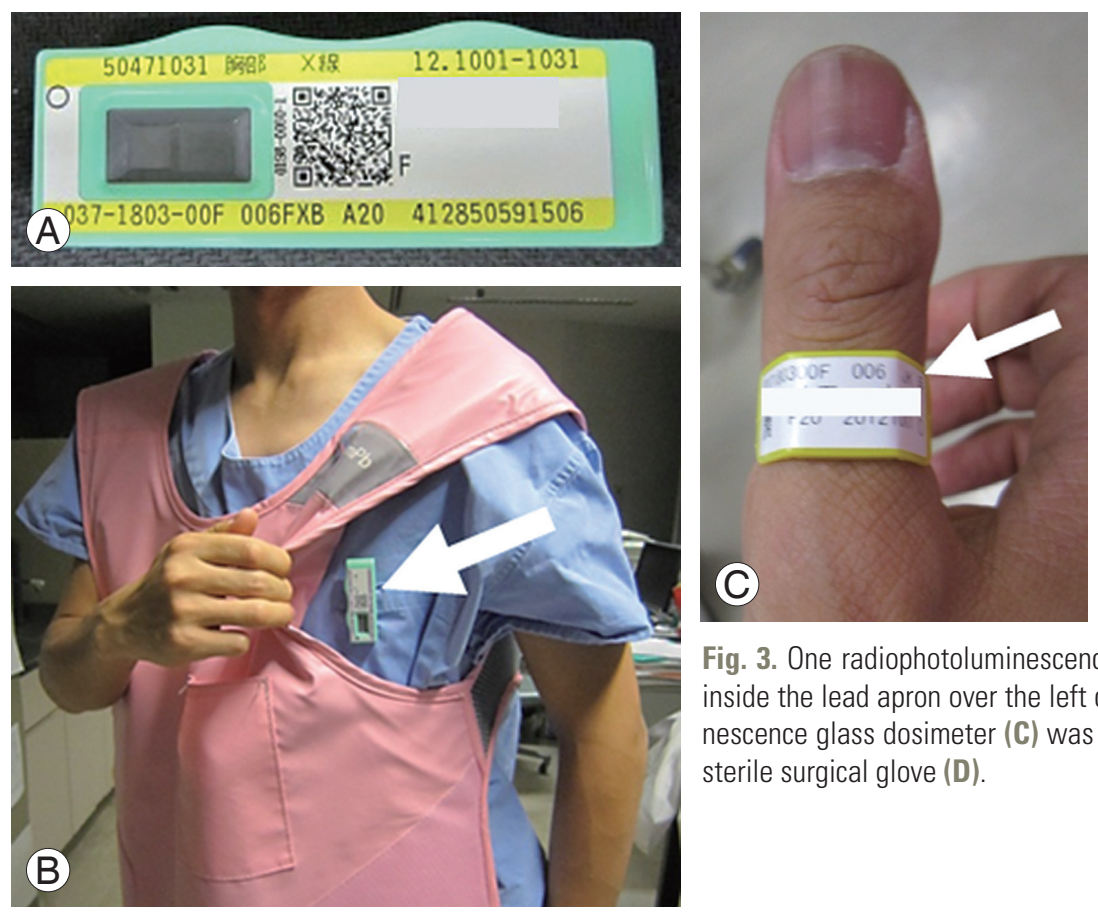

Fig. 3. One radiophotoluminescence glass dosimeter (A) was placed inside the lead apron over the left chest (B). One ring radiophotoluminescence glass dosimeter $(\mathrm{C})$ was worn on the right thumb under the sterile surgical glove (D). ray table (Fig. 4).

The steps of the fluoroscopic procedure of myelography were as follows. The patient was placed in the left lateral decubitus position, and cutaneous anesthesia was in-
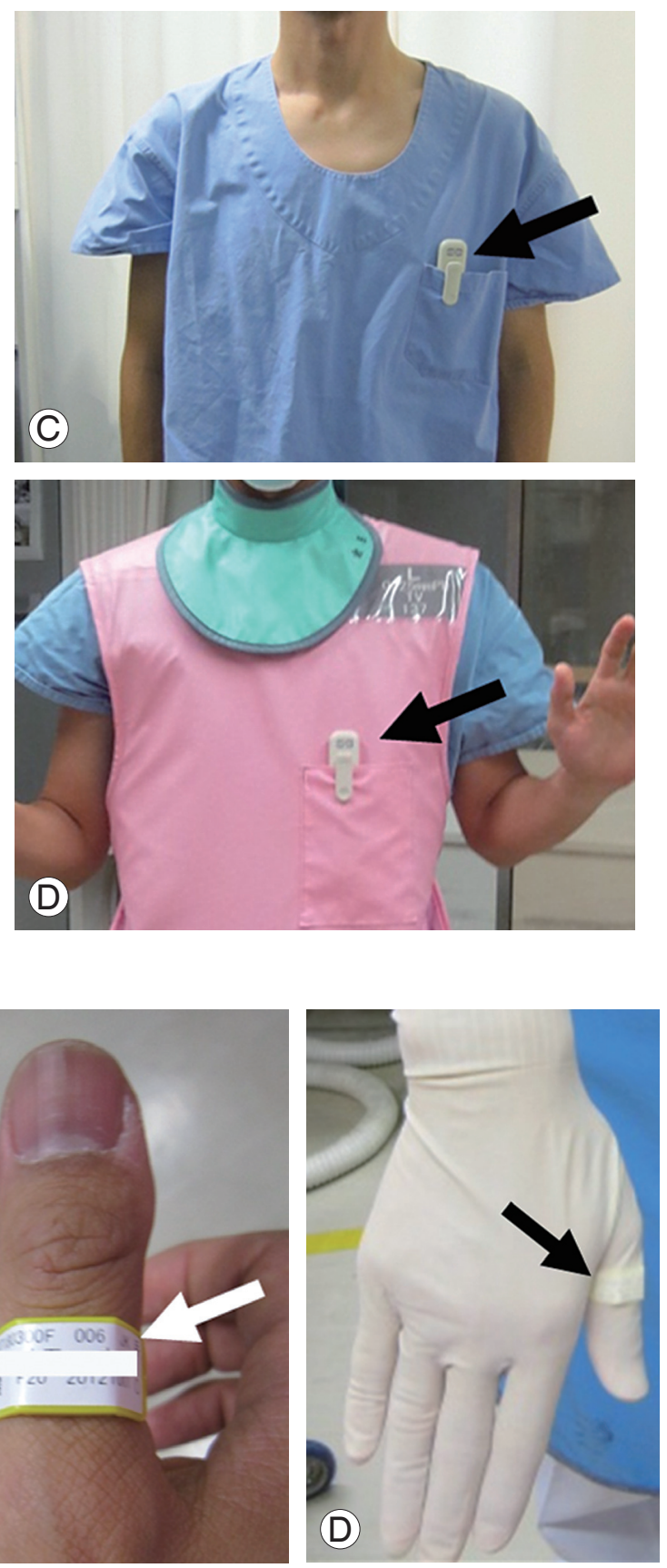

duced; lumbar puncture was then performed under aseptic conditions at the L2-3 or L3-4 level using a 22-gauge spinal needle. The lumbar needle was advanced through a midline approach under fluoroscopic guidance. Thereaf- 


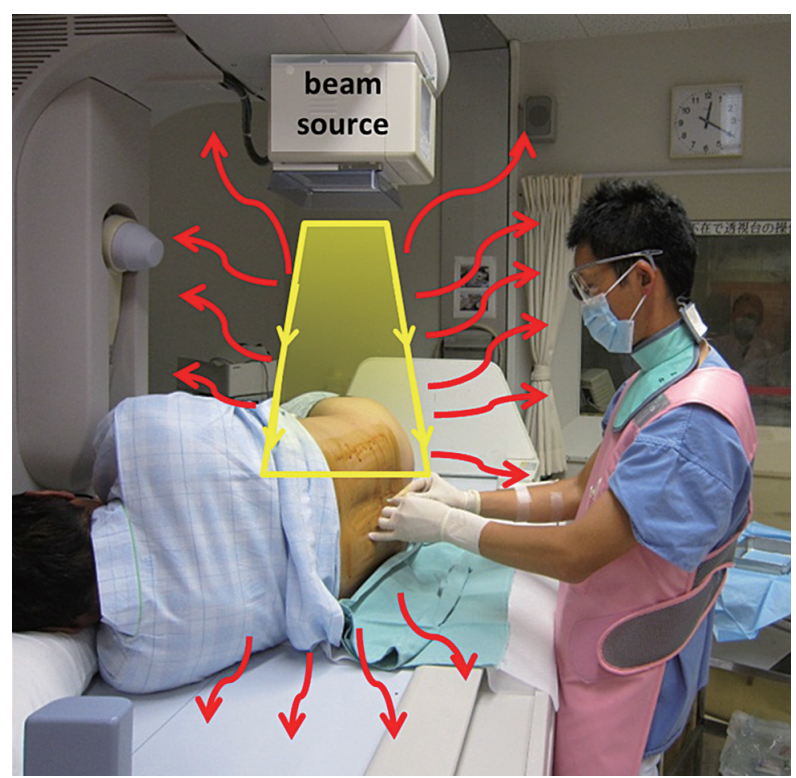

Fig. 4. Position of the spinal interventionalist, patient, and radiation beam source. The primary radiation and scattered radiation (curved arrows) to the spinal interventionalist.

ter, $10 \mathrm{~mL}$ of the contrast medium Iohexol (Omnipaque, Amersham Health, Princeton, NJ, USA) was injected slowly through the lumbar puncture needle. All myelograms were obtained in the lateral and prone positions, in both the anteflexion and retroflexion positions. Free flow of the contrast medium was noted up to the lower dorsolumbar region under fluoroscopy. Posteroanterior, lateral, and the $45^{\circ}$ right and left oblique views were obtained at the required levels. Radiographic images were also acquired in the standing position.

The following was the procedure for selective nerve root block and facet joint block. The injections were administered with the patient lying prone on the X-ray table. After sterile preparation, the puncture point was determined by obtaining an oblique fluoroscopic view of the specific nerve root or facet joint. Under repeated fluoroscopic screening, a 22-gauge spinal needle was advanced and positioned near the target nerve or facet joint. After injecting $1 \mathrm{~mL}$ of the contrast medium, we confirmed the correct placement of the needle and acquired posteroanterior and oblique view radiographs. Thereafter, $1 \mathrm{~mL}$ of $2 \%$ lidocaine was injected.

For each procedure, we obtained data regarding the duration of fluoroscopy and the amount of radiation exposure to the surgeon. Statistical analysis was performed using the Wilcoxon $t$-test. This study was approved by the Institutional Review Board of Kagawa Rosai Hospital (approval \#318).

\section{Results}

Sixteen patients underwent a fluoroscopic procedure in August, 18 in September, and 18 in October. Myelography was performed in 22 patients, myelography with selective nerve root block in 25 , selective nerve root block in 2 , and facet joint block in 3 .

The average duration of fluoroscopy per procedure was 3.7 minutes (range, 1.2-12.0 minutes). The average radiation exposure dose per procedure, as measured by the real-time dosimeters, was $14.9 \mu \mathrm{Sv}, 125.6 \mu \mathrm{Sv}$, and 200.1 $\mu \mathrm{Sv}$ inside the lead apron over the left chest (protected), outside the lead apron over the left chest (unprotected), and on the right forearm (unprotected), respectively (Fig. 5). As expected, to determine the effectiveness of the lead aprons, among the real-time dosimeters worn over the chest, the one worn inside the lead apron recorded significantly lower radiation doses than the unprotected one worn outside the lead apron. Importantly, the real-time dosimeter secured on the right forearm, which was held close to the X-ray beam, recorded significantly higher radiation doses than the unprotected real-time dosimeter worn outside the lead apron over the left chest.

Table 1 shows the radiation exposure doses recorded by the radiophotoluminescence glass dosimeter secured over the left chest inside the lead apron (protected) and the radiophotoluminescence glass ring dosimeter worn on the right hand thumb (unprotected). The protected radiophotoluminescence glass dosimeter over the left chest, which

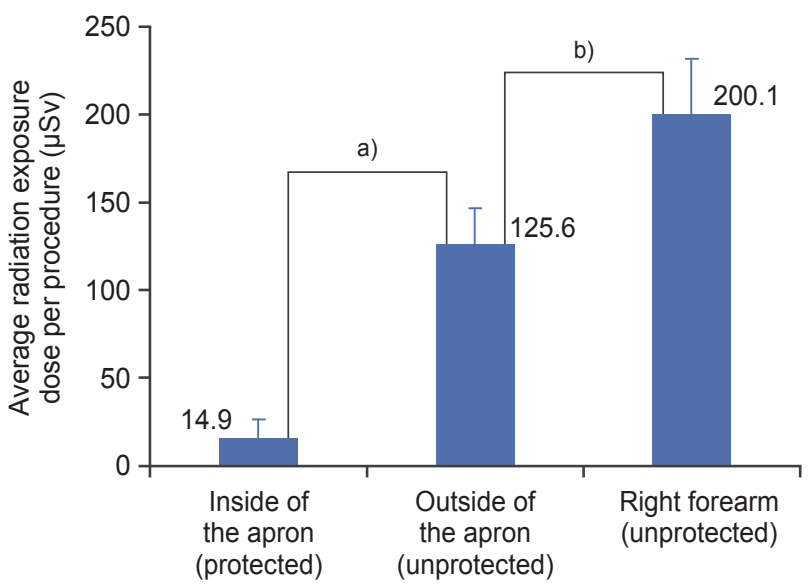

Fig. 5. Average radiation exposure dose for each real-time dosimeter per procedure. ${ }^{\text {a) }} p<0.001 ;{ }^{b)} p=0.007$ 
Table 1. Measurements made by the radiophotoluminescence glass dosimeter placed in front of the chest and the ring radiophotoluminescence glass dosimeter over 1 month

\begin{tabular}{lccc} 
Dosimeter & August & September & October \\
Chest, inside the apron (protected) & $\times$ & $\times$ & $\times$ \\
Right thumb (unprotected $m S v)$ & 122 & 120 & 126 \\
\hline
\end{tabular}

$x$, indicates that the dosimeter recorded less than the minimum reportable dose.

accumulated the effective whole-body dose for 1 month, recorded less than the minimum reportable dose in all the 3 months of the study period. On the other hand, the radiophotoluminescence glass ring dosimeter, which accumulated the radiation doses measured at the right thumb for each month, recorded $122 \mathrm{mSv}, 120 \mathrm{mSv}$, and $126 \mathrm{mSv}$ for August, September, and October, respectively.

According to the ICRP guidelines, the permissible annual individual radiation dose for the skin and extremities is $<500 \mathrm{mSv}$. In this study, the total of occupational radiation exposure doses received at the finger for the 3 months of the study period was $368 \mathrm{mSv}$ - extrapolated over a year, the annual radiation dose for the finger would be $1,472 \mathrm{mSv}$, which is almost three times the annual permissible limit.

\section{Discussion}

Orthopedic surgeons are exposed to ionizing radiation, both direct and scattered, during various procedures. Several studies have shown that the radiation doses during the use of a fluoroscope alone for various orthopedic procedures fall within the annual allowable dose for occupational radiation exposure $[15,16]$. However, the absolute exposure to orthopedic surgeons must be calculated on an individual basis, taking into account that radiation exposure is cumulative over a lifetime. At present, the long-term effects of low-dose radiation remain unknown [17].

In the case of orthopedic surgeons, the amount of radiation exposure cannot be kept at nil but it can be minimized as far as possible, according to the "as low as reasonably achievable" (ALARA) principle [18]. The cardinal principles applied for radiation protection are as follows: (1) maximize the distance from the radiation source, (2) use shielding materials, and (3) minimize exposure time.

The results of the current study showed that during spinal procedures, occupational radiation exposure to the upper extremity was significantly greater than that to the chest. In particular, with regard to the fingers, the extrapolated annual cumulative dose was considerably higher than the annual permissible dose limit stipulated by ICRP. This is because the hands and fingers of the interventionalist are often unavoidably exposed directly to the X-ray beam. Furthermore, the fluoroscopic device used by the interventionalist in this study was of the over-table type, where the X-ray beam is irradiated from top to bottom, leading to the hand and fingers being directly exposed to the X-ray beam without attenuation, in addition to the scattered radiation from the body of the patient. In the under-table type of fluoroscopic device, the X-ray beam is irradiated from the bottom to the top, which will substantially attenuate radiation exposure if the hand and fingers of the interventionalist stray into the path of the primary $\mathrm{X}$-ray beam.

In this study, the average duration of fluoroscopy per procedure was 3.7 minutes, which appears to be slightly long. This is because a slightly longer fluoroscopy duration was required to achieve clear contrast of the nerve root and facet joint. To shorten the duration of fluoroscopy, pulsed fluoroscopy or single-shot fluoroscopy should be used, rather than continuous fluoroscopy. Pulsed fluoroscopy reduces the overall exposure by $20 \%-75 \%[19,20]$.

The spinal interventionalist stood next to the patient throughout the procedure, performing the spinal procedure and instructing the patient about the position required to obtain clear radiographic images. Exposure to radiation decreases with an increase in the distance from the source. Given that the intensity of radiation at a point is inversely proportional to the square of distance, the hands of the interventionalist should be placed as far as possible from the irradiated part of the patient.

Statistical analysis showed that the radiation exposure inside the lead apron was significantly less than that outside it, indicating that the lead apron was effective in markedly reducing radiation exposure. With a view to reducing the amount of radiation exposure of the hands and fingers of the interventionalist during fluoroscopy, the 
effectiveness of lead gloves needs to be assessed in future studies. Mechlenburg et al. [21] reported that lead gloves showed poor absorption in their study. However, the manufacturer of the gloves claims that the gloves can attenuate radiation from primary X-ray beams of $100 \mathrm{kV}$ by $26 \%$, while also stating that the gloves are not intended for use in the path of or adjacent to the primary X-ray beam. The purpose of the gloves is to reduce the amount of scattered radiation exposure to the hands during fluoroscopy.

Future research needs to determine the benefits of collimation with regard to the radiation exposure of the hands and fingers of the interventionalist. All fluoroscopic equipment has the ability to collimate the beam, whereby the aperture can be reduced in size before the X-ray beam is emitted. Collimation reduces the beam area and enhances contrast, thereby decreasing the radiation exposure of the orthopedic surgeons [22].

This study has two limitations. First, the radiation exposure dose by the maneuver of each nerve root block and myelography alone are not differentiated. Second, the subject is only one person. However, this study revealed accurately the radiation exposure to the finger of a spinal interventionalist performing fluoroscopically guided spinal procedures.

To summarize, our study revealed that although the estimated effective whole-body annual dose received by the spinal interventionalist while performing spinal procedures is very low, radiation exposure to the dominant hand and fingers is likely to exceed the annual dose limit set by ICRP. Therefore, we recommend that spinal interventionalists take special care to note the position of their hands and fingers during fluoroscopy and make the necessary adjustments to limit the duration of fluoroscopy and radiation exposure. Additional epidemiologic studies are necessary to accurately determine the cumulative effect of low-dose radiation exposure from fluoroscopy to arrive at meaningful conclusions regarding lifetime exposure risks.

\section{Conclusions}

The cumulative radiation dose during fluoroscopically guided spinal procedures measured at the dominant hand may exceed the annual dose limit specified by the ICRP. Spinal interventionalists should take special care to limit the duration of fluoroscopy and radiation exposure.

\section{Conflict of Interest}

No potential conflict of interest relevant to this article was reported.

\section{References}

1. Frazier TH, Richardson JB, Fabre VC, Callen JP. Fluoroscopy-induced chronic radiation skin injury: a disease perhaps often overlooked. Arch Dermatol 2007;143:637-40.

2. Chambers JA, Long JN. Radiation injury and the hand surgeon. J Hand Surg Am 2008;33:601-11.

3. Liu X, Liu JZ, Zhang E, et al. Impaired wound healing after local soft $\mathrm{x}$-ray irradiation in rat skin: time course study of pathology, proliferation, cell cycle, and apoptosis. J Trauma 2005;59:682-90.

4. Brenner DJ, Doll R, Goodhead DT, et al. Cancer risks attributable to low doses of ionizing radiation: assessing what we really know. Proc Natl Acad Sci U S A 2003;100:13761-6.

5. Herscovici D Jr, Sanders RW. The effects, risks, and guidelines for radiation use in orthopaedic surgery. Clin Orthop Relat Res 2000;(375):126-32.

6. Chow R, Beaupre LA, Rudnisky CJ, Otto D, Clark M. Surgeons' perception of fluoroscopic radiation hazards to vision. Am J Orthop (Belle Mead NJ) 2013;42: 505-10

7. Mastrangelo G, Fedeli U, Fadda E, Giovanazzi A, Scoizzato L, Saia B. Increased cancer risk among surgeons in an orthopaedic hospital. Occup Med (Lond) 2005;55:498-500.

8. Chou LB, Chandran S, Harris AH, Tung J, Butler LM. Increased breast cancer prevalence among female orthopedic surgeons. J Womens Health (Larchmt) 2012;21:683-9.

9. Chou LB, Cox CA, Tung JJ, Harris AH, BrooksTerrell D, Sieh W. Prevalence of cancer in female orthopaedic surgeons in the United States. J Bone Joint Surg Am 2010;92:240-4.

10. Giordano BD, Grauer JN, Miller CP, Morgan TL, Rechtine GR 2nd. Radiation exposure issues in orthopaedics. J Bone Joint Surg Am 2011;93:e69(1-10).

11. Riley SA. Radiation exposure from fluoroscopy during orthopedic surgical procedures. Clin Orthop Relat Res 1989;(248):257-60.

12. Athwal GS, Bueno RA Jr, Wolfe SW. Radiation expo- 
sure in hand surgery: mini versus standard C-arm. J Hand Surg Am 2005;30:1310-6.

13. Giordano BD, Ryder S, Baumhauer JF, DiGiovanni $\mathrm{BF}$. Exposure to direct and scatter radiation with use of mini-c-arm fluoroscopy. J Bone Joint Surg Am 2007;89:948-52.

14. 1990 Recommendations of the International Commission on Radiological Protection. Ann ICRP 1991; 21:1-201.

15. Rampersaud YR, Foley KT, Shen AC, Williams S, Solomito M. Radiation exposure to the spine surgeon during fluoroscopically assisted pedicle screw insertion. Spine (Phila Pa 1976) 2000;25:2637-45.

16. Kruger R, Faciszewski T. Radiation dose reduction to medical staff during vertebroplasty: a review of techniques and methods to mitigate occupational dose. Spine (Phila Pa 1976) 2003;28:1608-13.

17. Adelstein SJ. Uncertainty and relative risks of radiation exposure. JAMA 1987;258:655-7.

18. National Council on Radiation Protection and Measurements. Implementation of the principle of as low as reasonably achievable (ALARA) for medical and dental personnel. NCRP report No.107. Bethesda: National Council on Radiation Protection and Measurements; 1990.

19. den Boer A, de Feyter PJ, Hummel WA, Keane D, Roelandt JR. Reduction of radiation exposure while maintaining high-quality fluoroscopic images during interventional cardiology using novel $\mathrm{x}$-ray tube technology with extra beam filtering. Circulation 1994;89:2710-4.

20. Hernandez RJ, Goodsitt MM. Reduction of radiation dose in pediatric patients using pulsed fluoroscopy. AJR Am J Roentgenol 1996;167:1247-53.

21. Mechlenburg I, Daugaard H, Soballe K. Radiation exposure to the orthopaedic surgeon during periacetabular osteotomy. Int Orthop 2009;33:1747-51.

22. Badman BL, Rill L, Butkovich B, Arreola M, Griend RA. Radiation exposure with use of the mini-C-arm for routine orthopaedic imaging procedures. J Bone Joint Surg Am 2005;87:13-7. 